\title{
Intraoperative Reproductive System Injury
}

National Cancer Institute

\section{Source}

National Cancer Institute. Intraoperative Reproductive System Injury. NCI Thesaurus.

Code C78387.

Damage to the reproductive organs during a surgical procedure. 